\title{
SJRM \\ A rare case of duodenal atresia with severe gastric bleeding, treated by vagotomy
}

\section{ARTICLE INFO}

\section{Article Type}

Case Report

\section{Authors \\ Seyyed Javad Seyyedi ${ }^{1}$, MD* Seyyed Arsalan Seyyedi ${ }^{1}$, MD Mohammad Aamin Samet ${ }^{1}$, MD Seyed Maziyar Mortazavi ${ }^{1}$, MD Maryam Sanaye Naderi² B.S \\ Mohammad Reza Nateghi ${ }^{2}$ (iD MD \\ ${ }^{1}$ Sarem Fertility and Infertility Research Center (SAFIR), Sarem Women's Hospital, Iran University of Medical Sciences (IUMS), Tehran, Iran \\ Sarem Fertility \& Infertility Research Center (SAFIR) \& Sarem Cell Research Center (SCRC), Sarem Women's Hospital, Iran University of Medical Sciences (IUMS), Tehran, Iran.}

*Corresponding Author

Address: Sarem Women Hospital, Basij Square, Phase 3, EkbatanTown, Tehran, Iran. Postal code:1396956111

Phone: +98 (21) 44670888

Fax: +98 (21) 44670432

dr.SEYYEDI@sarem.org

\section{Article History}

Received: May 04, 2020

Accepted: June 14, 2020

e Published: February 23, 2021

\section{ABSTRACT}

Introduction: Embryonic development of the gastrointestinal tract is a very complex process and therefore the number of congenital malformations of the fetus is reported to be numerous and varied. The most common site for atresia, after the esophagus, is the duodenum. Duodenal atresia is the congenital absence or complete blockage of a part of the duodenal lumen. This atresia is seen in perinatal examinations as polyhydramnios in pregnancy ultrasounds. Early detection of intestinal obstruction is essential to prevent further complications.

Patient Information: The present study is a case report of a 34-week-old preterm infant weighing 1980g, resulting from cesarean delivery with Apgar 8 and 9, who was diagnosed as duodenal atresia in prenatal examinations. The patient was a candidate for surgery on the day after birth, who suffered from severe gastric bleeding on the same day and was rushed to the operating room. In the operating room, due to severe bleeding and hypotension, with great effort by the anesthesiologist, the infant was intubated and subsequently, the infant underwent gastrotomy and blood clots were removed. There was oozing of blood from the mucosal surface. Then, duodenodenodenostomy surgery was performed, which continued after bleeding into the stomach, so in these conditions, several solutions such as total gastrectomy, pacing, and vagotomy were proposed. The infant underwent anterior-posterior truncal vagotomy. Gastric bleeding was controlled immediately after vagotomy. Because vagotomy can cause the pyloric constriction, gastrojejunostomy is also performed on the baby. The infant was discharged in good general condition without any complications after spending time in the neonatal intensive care unit.

Conclusion: Severe gastrointestinal bleeding is a very rare occurrence in duodenal atresia. In this situation, a gastrectomy can be considered and performed, which will be associated with many problems in the future. In adults, a vagotomy with a vascular contraction mechanism is used to control gastric bleeding. However, no case has been reported in the control of gastrointestinal bleeding in infants. In this case, for the first time, neonatal vagotomy was performed despite special technical subtleties, which was also accompanied by excellent therapeutic results.

Keywords: Fetal Gastrointestinal Anomalies, Duodenal Atresia, Duodenal Stenosis, Gastric Bleeding, Vagotomy, Duodenoduodenostomy, Case Report

Copyright(C) 2020, ASP Ins. This open-access article is published under the terms of the Creative Commons Attribution-Noncommercial 4.0 International License which permits Share (copy and distribute the material in any medium or format) and Adapt (remix, transform, and build 


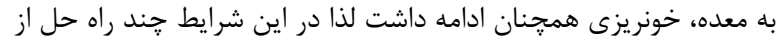

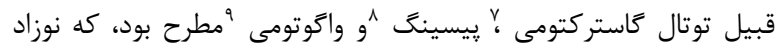

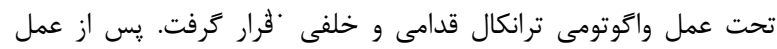

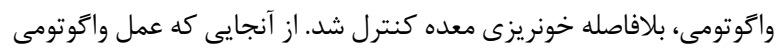

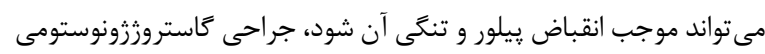

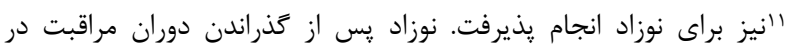

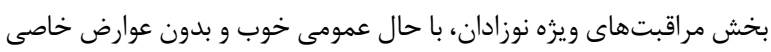
مرخص كرديد. بحث و نتيجه گيرى: خونريزى شديد گَوارشى در آترزى دئونوم اتفاقى بسيار نادر مىباشد. در مواجهه با جنين رخدادى كاستركتومى مىتواند

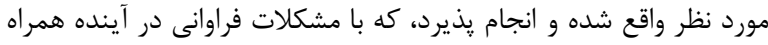

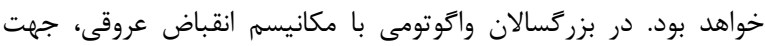

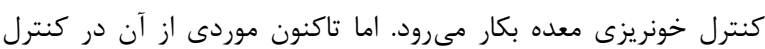

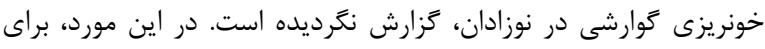

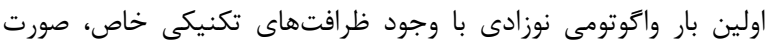

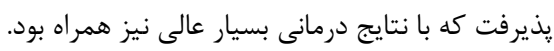

كليد وازمها: هاى گَوارشى جنينى، آترزى دئودنوم، تنكى دئودنوم،

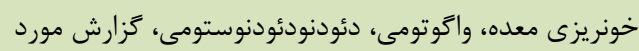

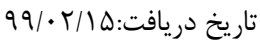

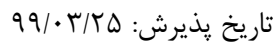

*ويسنده مسئول: سيد جواد سيدى تاريخ

مقدمه

نمو جنينى دستگاه گَارش فرآيندى بسيار پِيجيده مى باشد و به همين

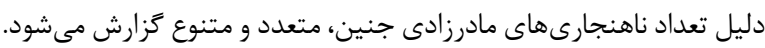

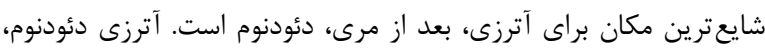

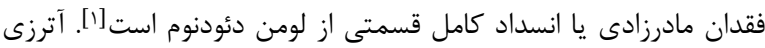

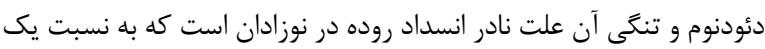

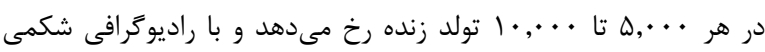

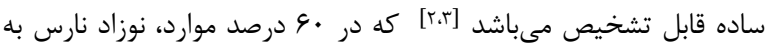

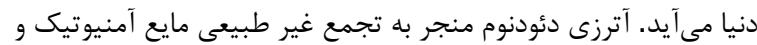

Total gastrectomy ${ }^{\mathrm{V}}$ Pacing ${ }^{\wedge}$ Vagotomy ${ }^{9}$ Anterior-Posterior Truncal Vagotomy' Gastrojejunostomy'

Total gastrectomy
Pacing $^{\wedge}$
Vagotomy
${ }^{\wedge}$
Anterior-Posterior Truncal Vagotomy
Gastrojejunostomy

يك مورد نادر آترزى دئودنوم همراه با خونريزى شديد معده، درمان شده

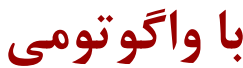

سيد جواد سيدى' *، سيد ارسلان سيدى '، محمد امين صامت '، سيد

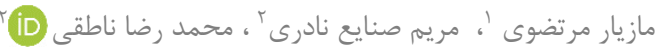
' مركز تحقيقات بارورى و نابارورى صارم، بيمارستان فوق تخصصى صارم،

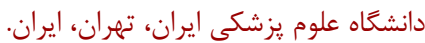

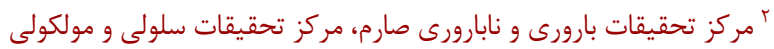

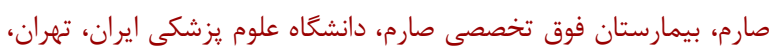

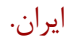

جكيده مقدمه: نمو جنينى دستخاه گوارش فرآيندى بسيار پِيجِيده مىباشد و به

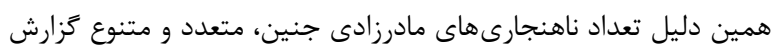

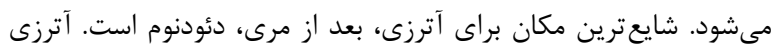

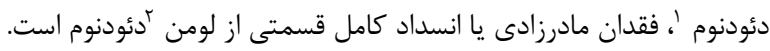

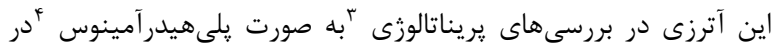

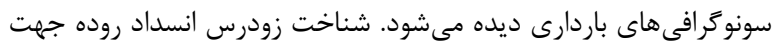

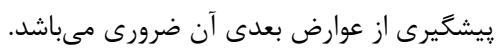

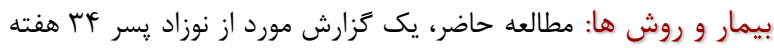

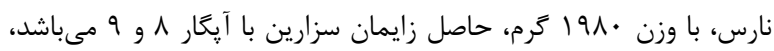

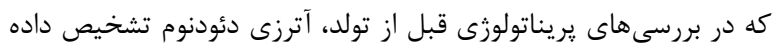

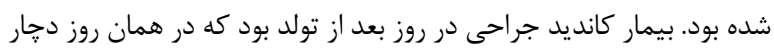

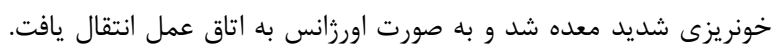
در اتاق عمل به دليل خونريزى شديد و افت فشار خون، با تلاش بـ بسيار

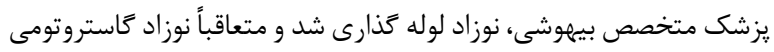

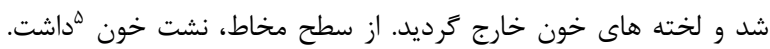

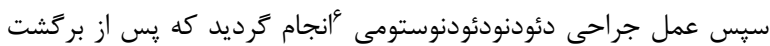

Duodenal Atresia Lumen' Perinatology Polyhydramnios Oozing Duodenoduodenostomy دانشنامه صارم در طب بارورى 


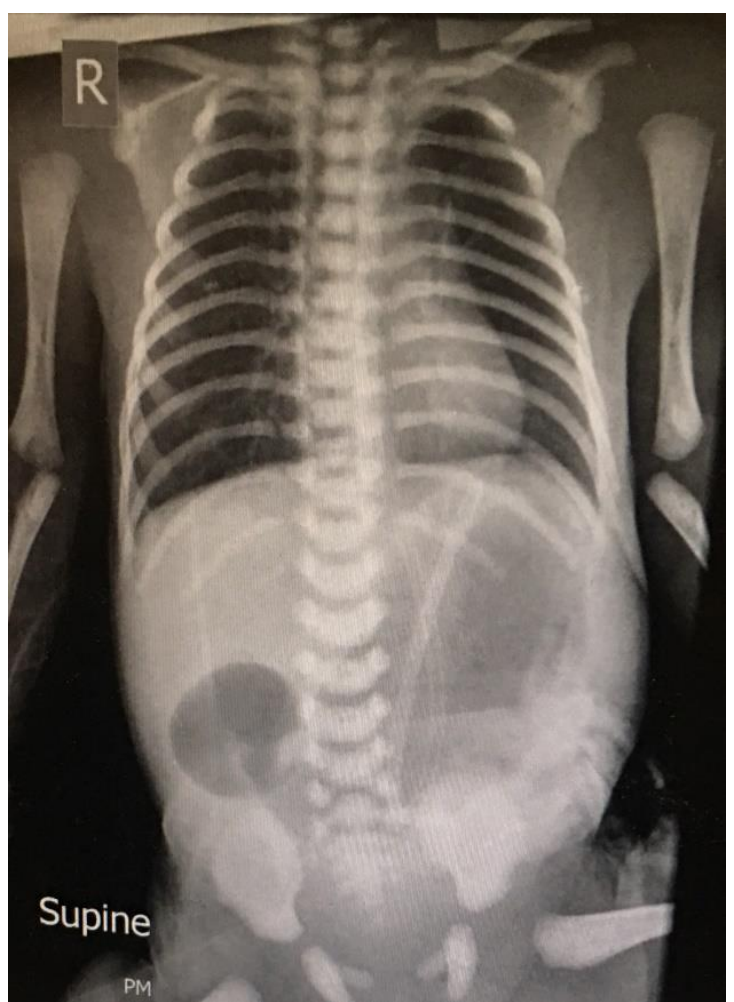

تصوير شماره ا: نماى حباب دوتايى در گرافى شكم نوزاد مشكوك به آترزى دئودنوم

مقرر كرديد نوزاد روز بعد از تولد، جهت برطرف كردن آترزى دئودونوم تحت

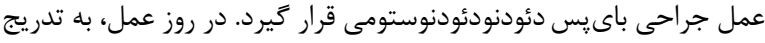

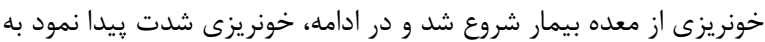

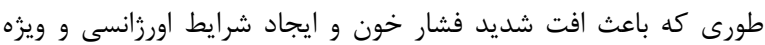

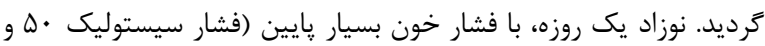

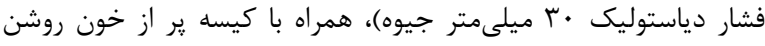

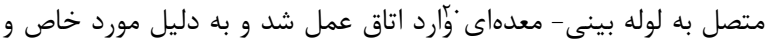

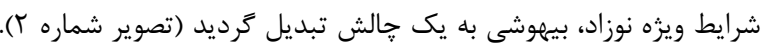

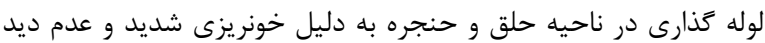

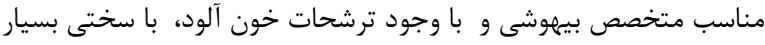

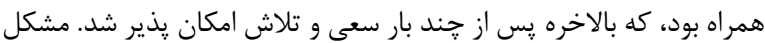

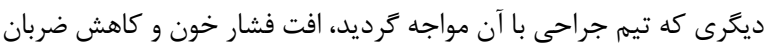

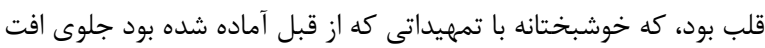

Duodenal Bulb Sign

Stenosis

Aneuploidy

Nasogastric Tube (NGT)
يلى هيدر آمنيوس مى شود كه با سونوگرافى قبل از تولد قابل تشخيص است.

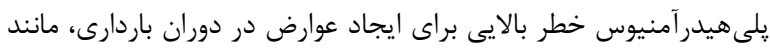

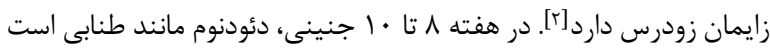

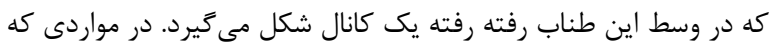

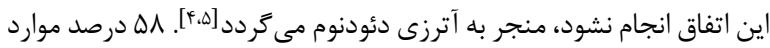

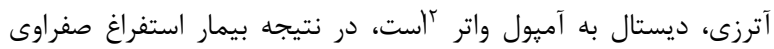

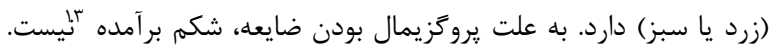

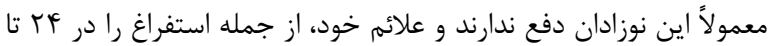

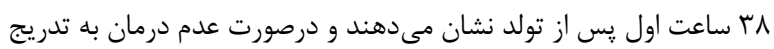

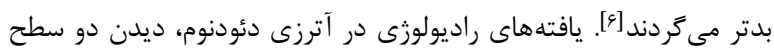

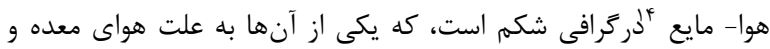

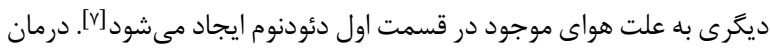

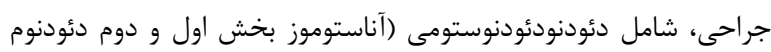

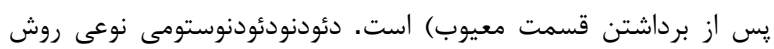

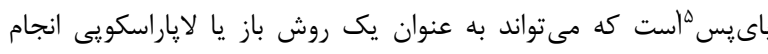
شود]ㅅ․]. مطالعه حاضر، كزارش يك مورد نادر از آترزى دئودنوم همراه با خونريزى

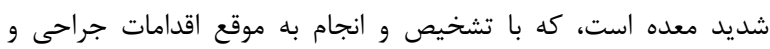

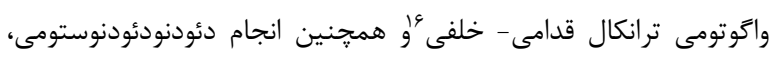

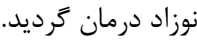

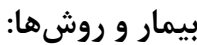

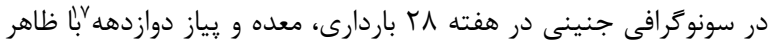

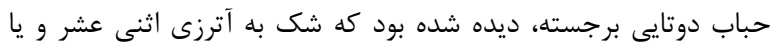

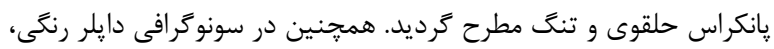

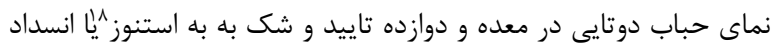

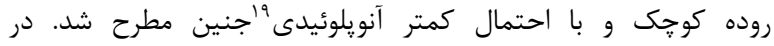

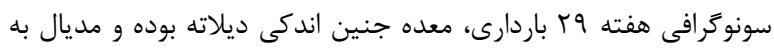

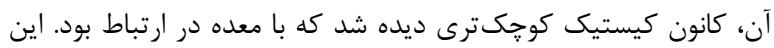

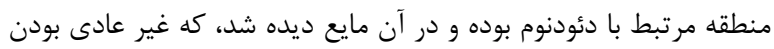

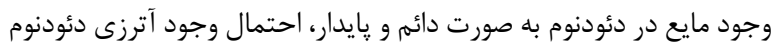

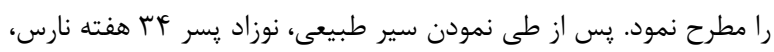

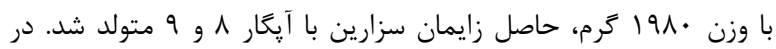

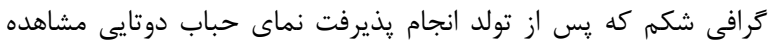

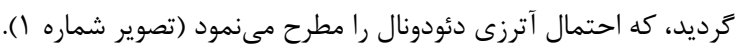


نارس بود و انجام توتال كاستر كتومى مىتوانست با عوارض بسيار يا حتى

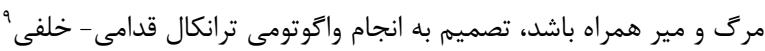

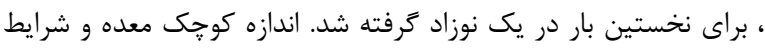

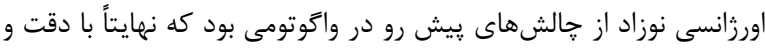

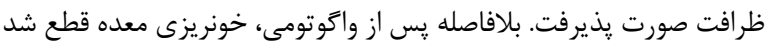

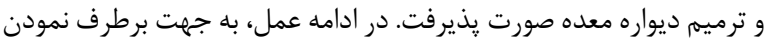

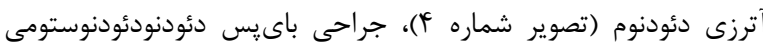

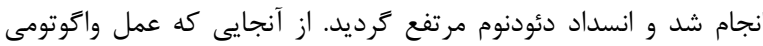

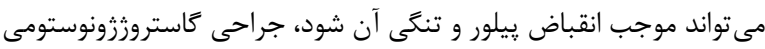

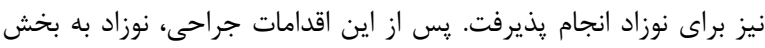

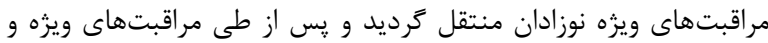

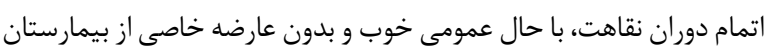

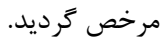

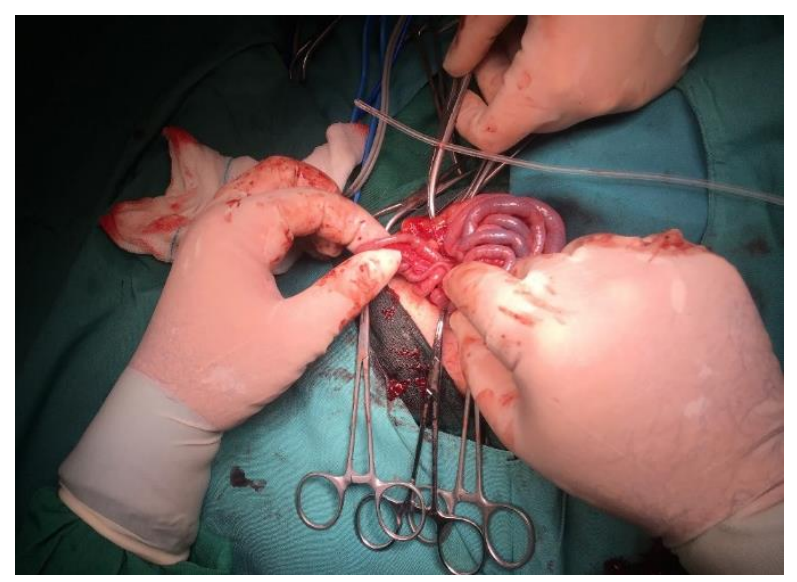

تصوير شماره٪: آترزى دئودنوم در نوزاد نارس يك روزه

در ويزيت سه ماه بعد، كودى در شرايط نرمال و خوبى معاينه شد و از نظر رشد و تكامل در وضعيت خوب و نرمالى بود. (تصوير شماره ه)
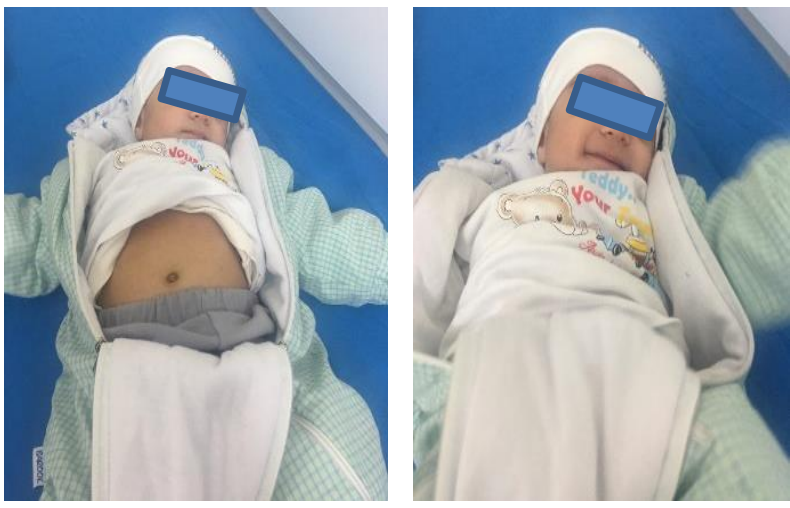

تصوير شمارهه: سه ماهكى كودى

Total Gastrectomy
فشار شديد و ايست قلبى گرفته شد و بيهوشى آغاز گرديد.

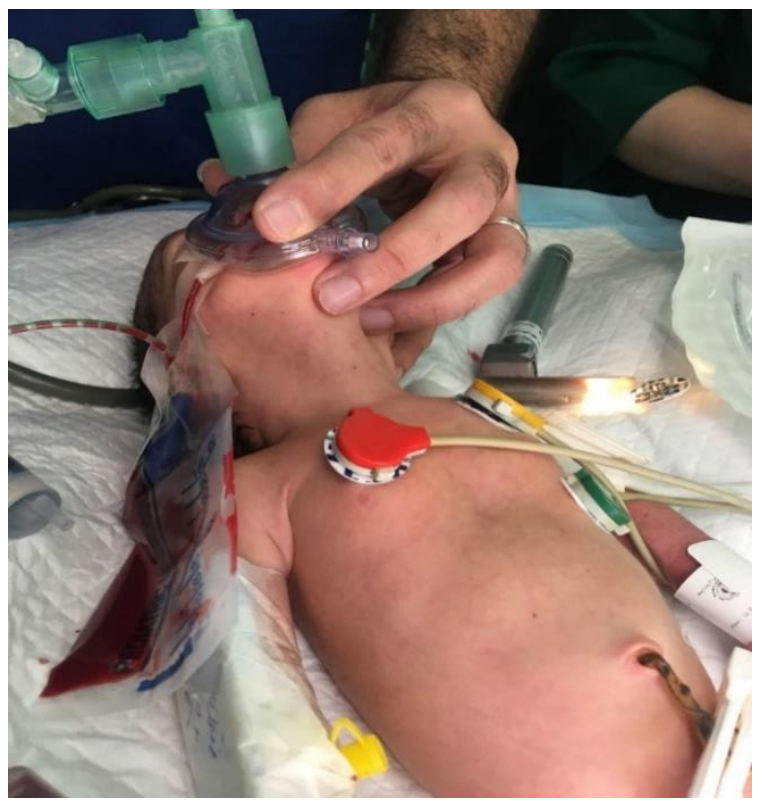

تصوير شماره厂: نوزاد با لوله بينى-معدهاى و كيسه ير از خون روشن (دقايقى بيش از جراحى)

يس از آماده سازى 'َّو بازكردن شكم، كاستروتومى 'آنجام شد، كه در

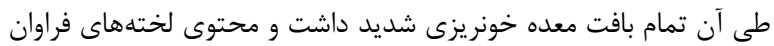

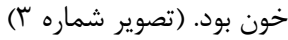

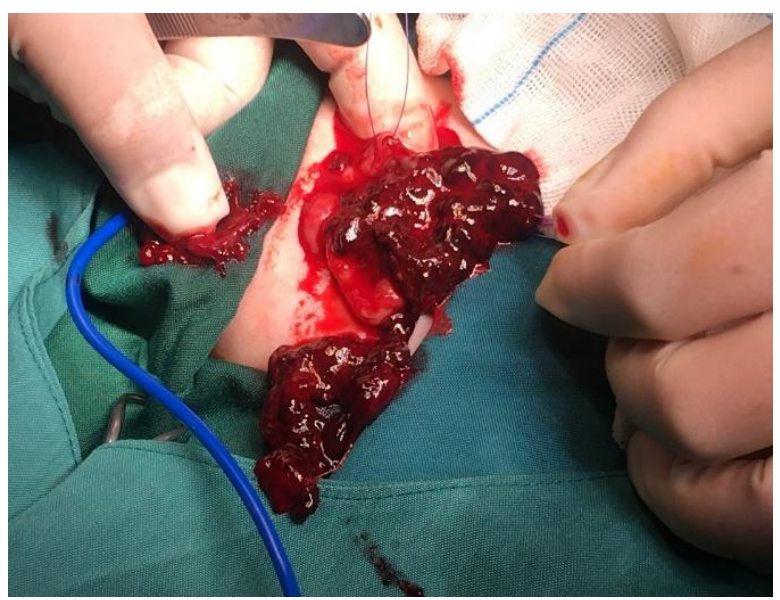

تصوير شماره ب: خروج لخته خون فراوان از معده نوزاد پِ از كاستروتومى

نتايج:

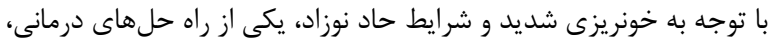

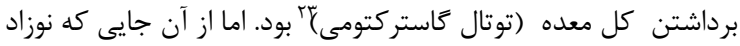

Prep. \& Drep. Gastrotomy

دانشنامه صارم در طب بارورى - م 
تشكر و قدردانى:

بدين وسيله از جناب آقاى دكتر ابوطالب صارمى و كاركنان محترم

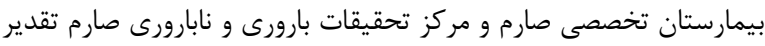

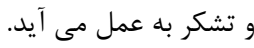

تاييديه اخلاقى:

اين طرح مورد تاييد كميته اخلاق (IEC) مركز تحقيقات بارورى و نابارورى (حسى

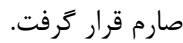

تعارض منافع:

در اين مطالعه تعارض منافع وجود نداشت.

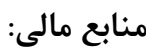

اين طرح با يشتيبانى مالى مركز تحقيقات بارورى و نابارورى صارم انجام

بذيرفت.

شناسه اركيد نويسند

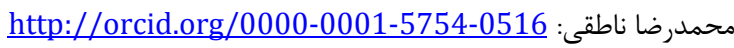

منابع

1- Bailey BV, Tracy TF, Connors RH, Mooney DP, Lewis JE, Weber TR. Congenital duodenal obstruction: a 32-year review. J Pediatr Surg 1993;28:92-5.

2- National Institutes of Health. Duodenal atresia. Updated 2018.

3- Morris JK, Springett AL, Greenlees R, Loane M, Addor MC, Arriola L, Barisic I, Bergman JEH, Csaky-Szunyogh M, Dias C, Draper ES, Garne E, Gatt M, Khoshnood B, Klungsoyr K, Lynch C, McDonnell R, Nelen V, Neville AJ, O'Mahony M, Pierini A, Queisser-Luft A, Randrianaivo $H$, Rankin J, Rissmann A, Kurinczuk J, Tucker D, Verellen-Dumoulin C, Wellesley D, Dolk H. Trends in congenital anomalies in Europe from 1980 to 2012. PLoS ONE. 2018;13(4):e0194986. [PMC free article] [PubMed]
آترزى دئودنوم به همراه خونريزى شديد گَوارشى بسيار نادر است. هنحام

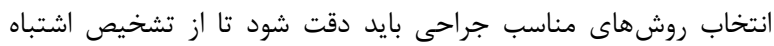

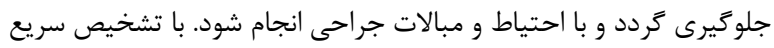

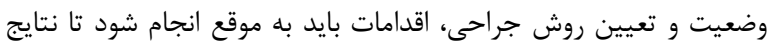

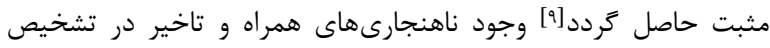

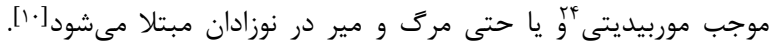

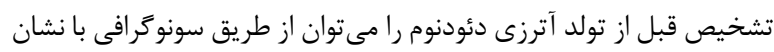

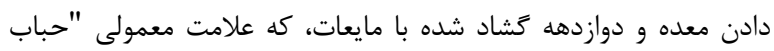

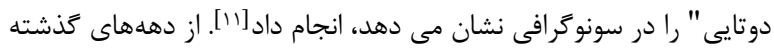

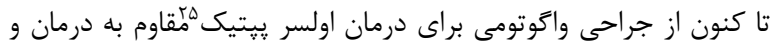

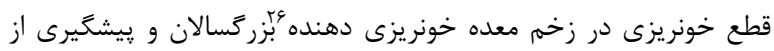

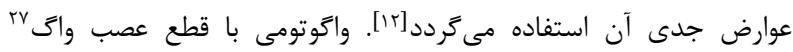

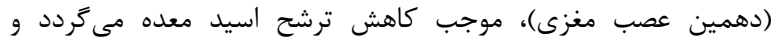

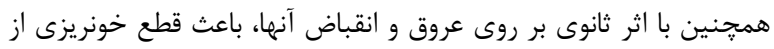

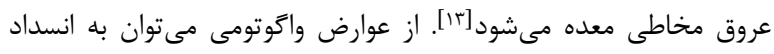

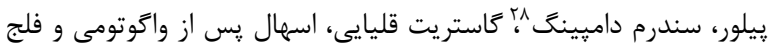

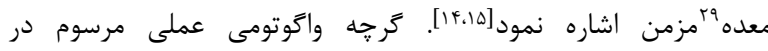

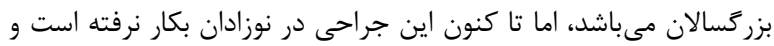

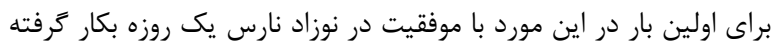

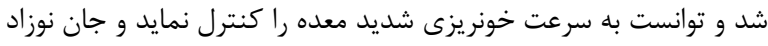

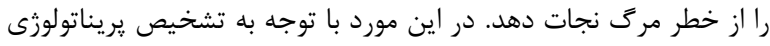

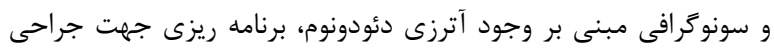

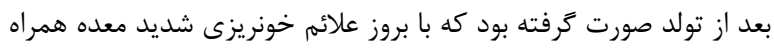

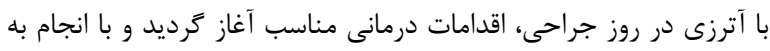

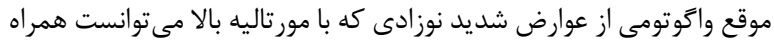

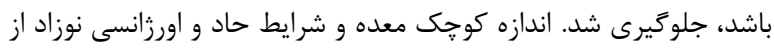

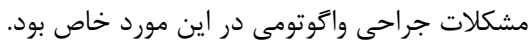

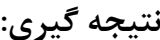

آترزى دئودنوم يك بيمارى نادر مادرزادى است كه در صورت عدم درمان

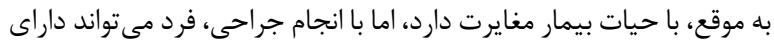

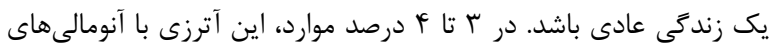

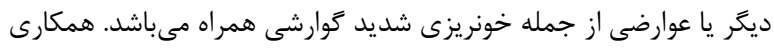

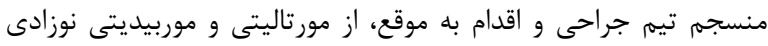
جلو ئيرى مىنمايد.
Vagus Nerve Dumping Syndrome Gastroparesis
Morbidity Peptic Ulcer Erosive Hemorrhagic Gastritis

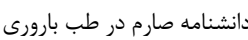


15- Seeras K, Qasawa RN, Prakash S. Truncal Vagotomy. [Updated 2020 Dec 18]. In: StatPearls [Internet]. Treasure Island (FL): StatPearls Publishing; 2020 Jan-. Available from: https://www.ncbi.nlm.nih.gov/books/NBK5261 04/
4- Sheorain VK, Cohen HL, Boulden TF. Classical wind sock sign of duodenal web on upper gastrointestinal contrast study. J Paediatr Child Health. 2013 May;49(5):416-7. [PubMed]

5- asad TR, Bajpai M. Intestinal atresia. Indian J Pediatr. 2000 Sep;67(9):671-8. [PubMed]

6- Gilet AG, Dunkin J, Cohen HL. Pylorospasm (simulating hypertrophic pyloric stenosis) with secondary gastroesophageal reflux. Ultrasound Q. 2008 Jun;24(2):93-6. [PubMed]

7- Koberlein G, DiSantis D. The "double bubble" sign. Abdom Radiol (NY). 2016 Feb;41(2):334-5. [PubMed]

8- Oh C, Lee S, Lee SK, Seo JM. Laparoscopic duodenoduodenostomy with parallel anastomosis for duodenal atresia. Surg Endosc. 2017 Jun;31(6):2406-2410. [PubMed]

9- Shuai Qiang, Meili Fan, Qingbo Cui, Zhaozhu Li, Yu Zhou, Qiang Li, Fengyong Li. Management of duodenal atresia associated with situs inversus abdominus: A case report. 2020 Jul 31;99(31): e21439.

10- J.A. Akinmoladun, T.A. Lawal, and A. Hafiz. LATE THIRD TRIMESTER ULTRASOUND DIAGNOSIS OF DUODENAL ATRESIA - THE IMPORTANCE OF DETAILED PRENATAL ULTRASOUND SCREENING. 2019 Jun; 17(1): 71-74.

11- Osifo OD. Duodenal Obstruction: Etiology, morbidity and mortality among Edo State Children. Nigeria. Afr J Biomed Res. 2009; 12(3):193-197.

12- Paul W.JohnstonM.D. William H.SnyderJr.M.D. Vagotomy and pyloroplasty in infancy and childhood. Journal of Pediatric Surgery. April 1968, Pages 238-245.

13- Gurusamy, K. S., \& Pallari, E. (2016). Medical versus surgical treatment for refractory or recurrent peptic ulcer. The Cochrane database of systematic reviews, 3(3), CD011523. https://doi.org/10.1002/14651858.CD011523. pub2

14- Schroder, V. T., Pappas, T. N., Vaslef, S. N., De La Fuente, S. G., \& Scarborough, J. E. (2014). Vagotomy/drainage is superior to local oversew in patients who require emergency surgery for bleeding peptic ulcers. Annals of surgery, 259(6), 1111-1118.

https://doi.org/10.1097/SLA.00000000000003 $\underline{86}$ 